\title{
A comparative analysis of tuberculosis in vitro screening in pemphigus patients selected for treatment with rituximab
}

\author{
Magdalena Jałowska', ${ }^{1,2}$ Justyna Gornowicz-Porowska', ${ }^{1,3}$ Agnieszka Seraszek-Jaros ${ }^{4}$, Elżbieta Kaczmarek ${ }^{4}$, \\ Monika Bowszyc-Dmochowska ${ }^{5}$, Paweł Bartkiewicz ${ }^{1}$, Marian Dmochowski ${ }^{1}$
}

\begin{abstract}
${ }^{1}$ Autoimmune Blistering Dermatoses Section, Department of Dermatology, Poznan University of Medical Sciences, Poznan, Poland 2Occupational Medicine Outpatient Clinic, Święcicki Hospital, Poznan University of Medical Sciences, Poznan, Poland 3Department and Division of Practical Cosmetology and Skin Diseases Prophylaxis, Poznan University of Medicinal Sciences, Poznan, Poland

${ }^{4}$ Department of Bioinformatics and Computational Biology, Poznan University of Medical Sciences, Poznan, Poland

${ }^{5}$ Cutaneous Histopathology and Immunopathology Section, Department of Dermatology, Poznan University of Medical Sciences, Poznan, Poland
\end{abstract}

Adv Dermatol Allergol 2021; XXXVIII (4): 611-614 DOI: https://doi.org/10.5114/ada.2021.108904

\begin{abstract}
Introduction: Patients qualified for the Polish government programme of treating severe pemphigus diseases with rituximab (RTX) available in 2018-2019 had to meet numerous criteria, including no active infectious disease. Aim: The clinical usefulness of tuberculosis screening with the QuantiFERON-TB Gold Plus (QFT-Plus) in native pemphigus patients selected for RTX treatment was statistically evaluated.

Material and methods: Eighteen pemphigus patients were examined with QFT-Plus prior to the intended RTX therapy. Ninety hospital employees examined with QFT-Plus due to contact with a cleaning worker who was diagnosed with active pulmonary tuberculosis were the control group.

Results: Six of 18 pemphigus patients had a positive QFT-Plus test result, one indefinite result and one initially indefinite and then negative. In the control group, 26 of 90 employees had a positive test result and none had an indefinite result. Statistical analysis by Fisher's exact test showed no statistically significant difference in QFT-Plus positive results between the groups $(p=0.5577)$. Only in 1 patient with recurrent mucocutaneous pemphigus vulgaris previously treated with traditional immunosuppression, lung changes were detected by computed tomography. No employee had any changes in the chest radiograph.

Conclusions: Prior immunosuppression and autoimmunity might be the cause of indefinite test results, but they do not seem to increase positive results. In the native population, the QFT-Plus screening reveals a significant population exposure to $M$. tuberculosis infection independent of pemphigus autoimmunity, and such screening can be a starting point for identifying patients requiring anti-tuberculosis drug prophylaxis before combined RTX-glucocorticosteroid treatment.
\end{abstract}

Key words: pemphigus, tuberculosis screening, rituximab.

\section{Introduction}

Pemphigus diseases are a group of potentially lifethreatening acantholytic illnesses of mucous membranes and/or skin. Pemphigus diseases are diagnosed on the basis of clinical manifestations, direct immunofluorescence of perilesional tissue, that can be visualized with various microscopy systems, for deposition of immunoreactants showing the pemphigus-pattern with, in case of lgG4, dew drops on spider web appearance and serum testing with molecular-biochemical techniques for autoantibodies to desmogleins [1-3]. Systemic glucocorticosteroids (GCS), alone or in combination with immunosuppressants or immunomodulation, are a long-established option for treating pemphigus diseases.

The programme of rituximab (RTX) treatment of severe immunosuppression-resistant pemphigus financed by

Address for correspondence: Prof. Marian Dmochowski MD, PhD, Autoimmune Blistering Dermatoses Section, Department of Dermatology, Poznan University of Medical Sciences, 49 Przybyszewskiego St, 60-355 Poznan, Poland, phone: +48 618691319 , e-mail: mdmochowski@ump.edu.pl Received: 26.12.2019, accepted: 12.03.2020. 
Table 1. QuantiFERON-TB Gold Plus test results and their statistical analysis in examined groups

\begin{tabular}{|c|c|c|c|}
\hline \multirow{2}{*}{$\begin{array}{l}\text { QuantiFERON-TB } \\
\text { Gold Plus } \\
\text { test results }\end{array}$} & \multicolumn{2}{|c|}{ Examined groups } & \multirow{2}{*}{$\begin{array}{c}\text { Statistical } \\
\text { analysis } \\
\text { of positive } \\
\text { results }\end{array}$} \\
\hline & $\begin{array}{l}\text { Pemphigus patients } \\
\text { selected for } \\
\text { rituximab }(n=18)\end{array}$ & $\begin{array}{l}\text { Hospital } \\
\text { employees } \\
(n=90)\end{array}$ & \\
\hline Positive & 6 & 26 & \multirow{4}{*}{$\begin{array}{c}\text { Fisher's } \\
\text { exact } \\
\text { test } \\
p=0.5577\end{array}$} \\
\hline Indefinite & 1 & 0 & \\
\hline $\begin{array}{l}\text { Initially indefinite } \\
\text { then negative }\end{array}$ & 1 & 0 & \\
\hline Negative & 10 & 64 & \\
\hline
\end{tabular}

a governmental agency functioned in Poland in 2018-2019. People eligible for this program were patients aged 18+ without an active infectious disease suffering from severe pemphigus resistant to immunosuppressive treatment as a principal indication. Main contraindications to the participation in the program included: age under 18, pregnancy, lactation period, hypersensitivity to the medicine's active substance or excipient, uncontrolled severe infections, including active hepatitis C and HIV infection, NYHA class IV circulatory insufficiency and severe immunodeficiency.

RTX is a chimeric monoclonal human-mouse antibody that has been used worldwide for many years for the treatment of pemphigus [4, 5]. It selectively binds to transmembrane antigen CD20 expressed on the surface of B lymphocytes and absent on other cells. RTX causes the lysis of cells containing CD20 antigen as a result of mechanisms dependent on both the complement system, and associated with antibody-dependent cellular cytotoxicity, and as a result of apoptosis.

Each pemphigus patient eligible for the participation in the programme underwent a whole set of tests, including QuantiFERON-TB Gold Plus (QFT-Plus) test used to exclude latent tuberculosis infection. This test is based on the evaluation of the quantity of interferon- $\gamma$ (IFN- $\gamma$ ) produced by $T$ lymphocytes as a result of their stimulation with ESAT- 6 and CFP 10 antigens by ELISA method [6].

\section{Aim}

There has been made a comparative assessment of the clinical usefulness of QFT-Plus test measuring in vitro the secretion of interferon $\gamma$ (IGRA) in native pemphigus patients examined with this test prior to the intended RTX therapy.

\section{Material and methods}

Eighteen pemphigus patients (the study group) were selected in the Autoimmune Blistering Dermatoses Section, Department of Dermatology, the Karol Marcinkowski Poznan University of Medical Sciences for QFT-Plus (Qiagen, Hilden, Germany) test prior to the intended RTX treatment. There were 15 pemphigus vulgaris patients (11 females and 4 males) and 3 pemphigus foliaceus patients ( 1 female and 2 males). The age range of the patients was $30-84$ years. The control group included 90 hospital employees tested with QFT-Plus in the Occupational Medicine Outpatient Clinic due to contact with a cleaner with diagnosed active pulmonary tuberculosis.

\section{Statistical analysis}

The statistical analysis of positive QFT-Plus test results was done with Fisher's exact test using Statistica, version 13 (StatSoft, Inc, Tulsa, OK, USA). A $p<0.05$ was arbitrarily considered statistically significant.

\section{Results}

In 6 of 18 (33.33\%) pemphigus patients the test result was positive, in 1 the result was indefinite, and in $1 \mathrm{ini}$ tially indefinite, and then negative. In 26 of 90 (28.88\%) hospital employees the test result was positive, in none of them the result was indefinite. The statistical analysis of positive QFT-Plus test results with Fisher's exact test revealed no statistically significant difference between both groups ( $p=0.5577$ ) (Table 1$)$.

In 1 patient, with recurring mucocutaneous pemphigus vulgaris (mcPV) (Figure 1) previously treated with traditional immunosuppression, the QFT-Plus test result was positive and lung lesions were shown in chest X-ray, in the form of a $7 \mathrm{~mm}$ oval shade in the left central lung area, and an oval shade of $8 \mathrm{~mm}$ diameter in the left lung apex. Fibrotic and tumour lesions in the left lung were shown in lung computed tomography (CT). The patient was not treated with RTX. She was referred for further treatment at the Outpatient Pulmonary Clinic.

Among the patients with originally non-negative test result, 1 patient with a positive result and with persistent mcPV coexisting with idiopathic thrombocytopenic purpura, after preventive administration of isoniazid + rifampicin for 6 months by the pulmonologist, was treated with RTX which resulted in normalisation of the thrombocytes count and temporary healing of mucosal lesions. The patient's chest X-ray performed before and after chemo-prophylactic treatment has shown no abnormalities. The patient did not report any problems with the pulmonary system, in the form of dyspnoea or cough. In the remaining 7 patients having initially nonnegative results, an initial decision was to refrain from RTX treatment.

All persons in the control group of the medical staff, both with the positive and the negative result of QFTPlus, had a comprehensive medical history check with regard to the manifestations of active tuberculosis done and chest X-ray performed. Persons with a positive QFTPlus result underwent additional pulmonary consultations. None of the persons reported subfebrile conditions, night sweat, cough, or fatigue. In none of the patients, 
deviations in chest X-ray view were shown. Those persons were not administered preventive pharmacotherapy after the pulmonary consultation.

\section{Discussion}

Among many contagious diseases, tuberculosis still remains one of the most frequent causes of sicknesses and deaths in the world [6]. It is estimated that one third of global population is infected with Mycobacterium tuberculosis, and most of the cases have the form of the latent tuberculosis infection. Most of the reported tuberculosis cases in Europe come from Romania, Poland, and the United Kingdom. Nowadays, humanity faces the challenge of drug resistant tuberculosis [7].

The described risk factors for the Mycobacterium tuberculosis infection being transformed into an active disease include, among other things, immunosuppressive treatment and systemic glucocorticosteroids (GCS) therapy [8]. Chronic GCS therapy increases the risk of primary infection with tuberculosis mycobacterium and reactivation of latent tuberculosis [9]. Usually, the course of tuberculosis infection in those patients is more severe than in the general population, taking even the form of miliary tuberculosis [9]. GCS medications inhibit immune response of both $\mathrm{T}$ - and $\mathrm{B}$-cells. They decrease the level of cytokines: Interleukin (IL) 1 (IL-1), IL-2, IL-3, IL-4, IL-5, $\mathrm{IL}-8$ and IFN- $\gamma$. They reduce proliferation of $\mathrm{T}$ lymphocytes, and induce their apoptosis, reduce the production of antibodies by B lymphocytes. Other immunosuppressive therapies increasing the risk of tuberculosis infection include: azathioprine, dose $\geq 2 \mathrm{mg} / \mathrm{kg} /$ day, methotrexate, dose $\geq 25 \mathrm{mg} /$ week, anti-TNF- $\alpha$ [9].

Chan and Yosipovitch [10] established that taking systemic GCS even in relatively low oral doses, by patients with dermatoses (pemphigus diseases, dermatomyositis) increases the probability of reactivation of latent tuberculosis or primary infection with tuberculosis. Therefore, all dermatological patients undergoing chronic treatment with GCS medications should undergo periodic screening tests for tuberculosis infection.

In this study the statistical analysis has not demonstrated any significant difference in positive results between pemphigus patients and medical staff members, which may indicate that the immunosuppressants used in patients and their autoimmune disease did not increase the probability of tuberculosis incidence. Both in the group of patients with pemphigus and in the group of hospital staff, the percentage of persons with positive QFT-Plus results was around 30\%. Similar results were obtained in the doctoral thesis by Gruszczyński, in persons with other pulmonary disease than tuberculosis; QUANTIFERON-TB GOLD IN TUBE result was positive in $29 \%$ of persons [11]. In our study, QFT-Plus test reveals significant exposure of the native population to the Mycobacterium tuberculosis infection, regardless of the
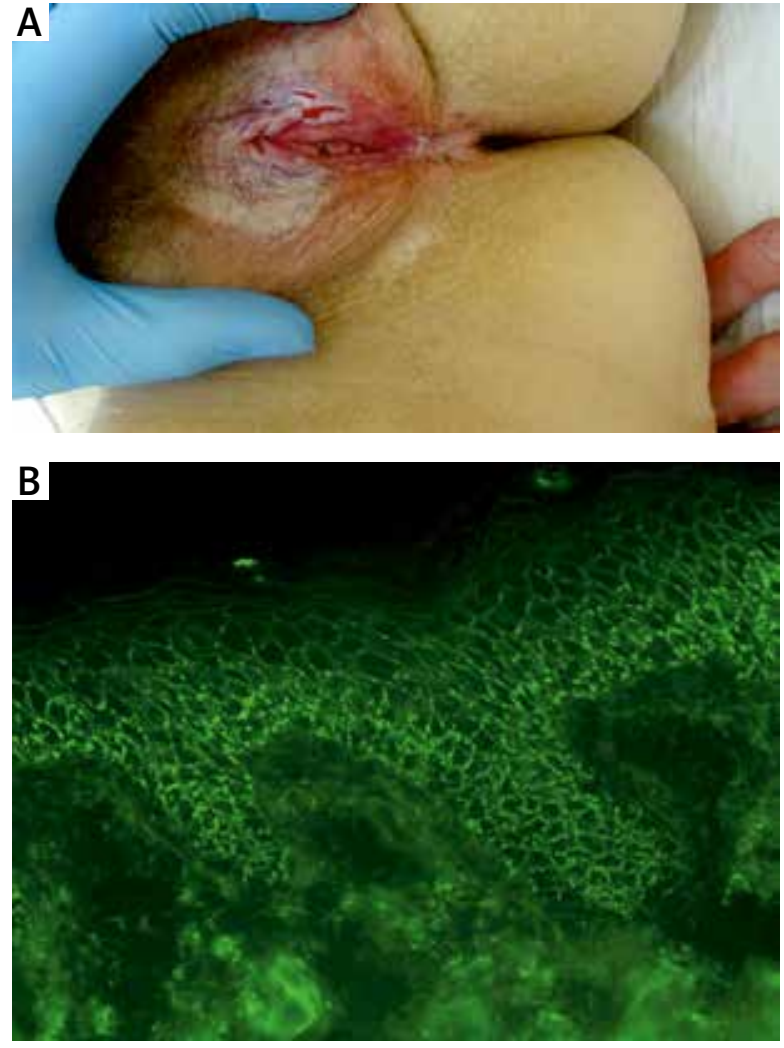

Figure 1. A middle-aged female with relapsing mucocutaneous pemphigus vulgaris having lesions around natural body orifices including erosions on the vulva (A). Direct immunofluorescence of perilesional skin visualized with the blue light-emitting diode technology-operated microscopy showed pemphigus IgG4(++) intercellular deposits having dew drops on spider web appearance (original objective magnification 40×) (B) and multi-analyte ELISA revealed elevated levels of serum anti-DSG1 (of 1.68), anti-DSG3 (of 5.2) and anti-envoplakin (of 1.86) IgG antibodies (cut-off ratio =1)

pemphigus autoimmunity, and its result may be the starting point for detection of patients requiring pharmaceutical prophylaxis against tuberculosis prior to the application of RTX combined with GCS.

As biological treatment with tumor necrosis factor $\alpha$ (TNF- $\alpha$ ) inhibitors usually causes activation of the latent form of tuberculosis (transition from latent to active form), candidates for biological treatment are diagnosed for mycobacterium infection, and treated, even for latent forms of the disease, in order to prevent the development of active tuberculosis. However, despite the negative test results of the presence of tuberculosis mycobacterium infection before the treatment, the patients may develop tuberculosis. Therefore, it seems relevant to repeat the tests in patients during the treatment. In the prospective study the data of 70 patients with rheumatic diseases (33 with rheumatoid arthritis, 33 with spondyloarthritis and 4 with other diagnosis) were analysed, in which, before the inclusion of medication from 
the group of TNF- $\alpha$ inhibitors, the tests for tuberculosis infection were negative (chest $X$-ray, IGRA tests and tuberculin sensitivity test). For a year the patients used different TNF- $\alpha$ inhibitors: adalimumab, etanercept, infliximab, golimumab, and certolizumab. After that period screening tests for tuberculosis infection were performed again. Positive results were obtained in 20 (29\%) patients. The tuberculin sensitivity test was positive in 9 (10\%) patients, T-SPOT.TB test was positive in $7(10 \%)$ patients, and QFT-GIT test was positive in $5(7 \%)$ patients. Only in 1 patient two tests gave positive results. In $40 \%$ of patients with the conversion of the tuberculosis test it was decided that a prophylactic treatment with anti-mycobacterium medication (isoniazid) should be used. During the follow-up period, lasting on average $27 \pm 12$ months, none of the patients has developed an active form of tuberculosis [12].

In case of rheumatoid arthritis treatment with RTX $(2 \times 1000 \mathrm{mg})$, the studies confirmed the occurrence of severe infections in comparison to persons receiving placebo, however, the increased frequency of tuberculosis incidence was not proven $[13,14]$. Alkadi et al. studied the influence of RTX on new tuberculosis infection and reactivation of latent tuberculosis in patients treated with RTX due to rheumatoid arthritis. The authors considered the screening test for tuberculosis prior to the inclusion of rituximab treatment pointless as the immune system uses cellular immunity to fight tuberculosis, whereas rituximab disables humoral immunity [15]. However, due to our patients' prior therapy with high doses of GCS before administering RTX, and the necessity to use GCS at the maintenance doses after RTX treatment as the response to of RTX may be delayed for months [16], the screening tests for tuberculosis seem to be fully justified.

\section{Acknowledgments}

A part of this study was presented at the Congress of the Youth Forum Section of the Polish Dermatological Society (24-25.10.2019, Lodz, Poland) and published as an abstract in Polish: Bartkiewicz P, Jałowska M, GornowiczPorowska J, Bowszyc-Dmochowska M, Seraszek-Jaros A, Kaczmarek E, Dmochowski M. Ocena porównawcza przydatności badania przesiewowego w kierunku gruźlicy testem in vitro mierzącym wydzielanie interferonu gamma u rodzimych chorych z pęcherzycą kwalifikowanych do leczenia rytuksymabem. Forum Dermatologicum 2019; 5: 138. This work was also undertaken in conjunction with activities of the European Reference Network-Skin.

\section{Conflict of interest}

The authors declare no conflict of interest.

\section{References}

1. Dmochowski M. Naturalne otwory ciała i błony śluzowe w pęcherzycy zwykłej. Dermatol Klin 2007; 9: 124-31.
2. Dmochowski M, Gornowicz-Porowska J, Bowszyc-Dmochowska M. Dew drops on spider web appearance: a newly named pattern of IgG4 deposition in pemphigus with direct immunofluorescence. Adv Dermatol Allergol 2017; 24: 1-4.

3. Gornowicz-Porowska J, Bowszyc-Dmochowska M, RaptisBolwach M, et al. Blue light-emitting diode technology-operated microscopy is preferable to both short arc mercury lamp-operated microscopy and laser scanning confocal microscopy for direct immunofluorescence images evaluation in routinely diagnosing subepidermal autoimmune blistering diseases. Microsc Res Tech 2019; 82: 1735-40.

4. Frampton JE. Rituximab: a review in pemphigus vulgaris. Am J Clin Dermatol 2020; 21: 149-56.

5. Mohamad AF, Iversen L, Bech R. Pemphigus vulgaris: short time to relapse in patients treated in a Danish tertiary referral center. Front Med 2019; 6: 259.

6. Borkowska D, Radzikowska E, Załęska J, et al. Interferongamma release assays in the diagnosis of latent tuberculosis infection in clinical situations. Pneumonol Alergol Pol 2014; 81: 39-45.

7. Gupta-Wright A, Tomlinson GS, Rangaka MX, Fletcher HA. World TB Day 2018: the challenge of drug resistant tuberculosis. F1000Research 2018; 7: 217.

8. Jick SS, Lieberman ES, Rahman MU, et al. Glucocorticoid use, other associated factors, and the risk of tuberculosis. Arthritis Rheum 2006; 55: 19-26.

9. Butt G, Faria A, Ijaz H. Pulmonary tuberculosis in dermatological patients on high-dose, long-term steroid therapy. J Pakistan Assoc Dermatol 2015; 15: 119-31.

10. Chan YC, Yosipovitch G. Suggested guidelines for screening and management of tuberculosis in patients taking oral glucocorticoids - an important but often neglected issue. J Am Acad Dermatol 2003; 49: 91-5.

11. Gruszczyński P. Evaluation of significance of QUANTIFERONTB GOLD IN TUBE diagnostic test for identifying Mycobacterium tuberculosis pulmonary infections/Ocena znaczenia testu diagnostycznego QUANTIFERON-TB GOLD IN TUBE w rozpoznawaniu zakażeń płucnych Mycobacterium tuberculosis. Ph.D. thesis. Poznan University of Medical Sciences, Poznan, 2011, 1-93.

12. Hatzara C, Hadziyannis E, Kandili A, et al. Frequent conversion of tuberculosis screening tests during anti-tumour necrosis factor therapy in patients with rheumatic diseases. Ann Rheum Dis 2015; 74: 1848-53.

13. Emery P, Fleischmann R, Filipowicz-Sosnowska A, et al. The efficacy and safety of rituximab in patients with active rheumatoid arthritis despite methotrexate treatment: results of a phase IIB randomized, double-blind, placebo-controlled, dose-ranging trial. Arthritis Rheum 2006; 54: 1390-400.

14. Cohen SB, Emery P, Greenwald MW, et al. Rituximab for rheumatoid arthritis refractory to anti-tumor necrosis factor therapy: results of a multicenter, randomized, double-blind, placebo-controlled, phase III trial evaluating primary efficacy and safety at twenty-four weeks. Arthritis Rheum 2006; 54 : 2793-806

15. Alkadi A, Alduaiji N, Alrehaily A. Risk of tuberculosis reactivation with rituximab therapy. Int J Health Sci 2017; 11: 41-4.

16. Bartkiewicz P, Gornowicz-Porowska J, Bowszyc-Dmochowska $M$, et al. No response to rituximab in a case of mucosaldominant to and fro mucocutaneous shifting pemphigus vulgaris previously treated with a range of more traditional anti-pemphigus therapies: clinical hint relevant to pemphigus pathogenesis. PPP 2016 - Pathogenesis of Pemphigus and Pemphigoid, The Open Blister/Mind Meeting. Satellite Symposium to the 46th Annual ESDR Meeting. Munich, Germany, 5-7 September 2016, Program and abstracts book, p. 39. 